\title{
Efficient Deep Learning Model for Mitosis Detection using Breast Histopathology Images
}

\author{
Monjoy Saha ${ }^{1}$, Chandan Chakraborty ${ }^{+, 1}$, Daniel Racoceanu ${ }^{2, *}$
}

\begin{abstract}
Mitosis detection is one of the critical factors of cancer prognosis, carrying significant diagnostic information required for breast cancer grading. It provides vital clues to estimate the aggressiveness and the proliferation rate of the tumour. The manual mitosis quantification from whole slide images is a very laborintensive and challenging task. The aim of this study is to propose a supervised model to detect mitosis signature from breast histopathology WSI images. The model has been designed using deep learning architecture with handcrafted features. We used handcrafted features issued from previous medical challenges MITOS @ ICPR 2012, AMIDA-13 and projects (MICO ANR TecSan) expertise. The deep learning architecture mainly consists of five convolution layers, four max-pooling layers, four rectified linear units (ReLU), and two fully connected layers. ReLU has been used after each convolution layer as an activation function. Dropout layer has been included after first fully connected layer to avoid overfitting. Handcrafted features mainly consist of morphological, textural and intensity features. The proposed architecture has shown to have an improved $92 \%$ precision, $88 \%$ recall and $90 \%$ F-score. Prospectively, the proposed model will be very beneficial in routine exam, providing pathologists with efficient and - as we will prove - effective second opinion for breast cancer grading from whole slide images. Last but not the least, this model could lead junior and senior pathologists, as medical researchers, to a superior understanding and evaluation of breast cancer stage and genesis.

Keywords: Breast cancer, mitosis, deep neural network, handcrafted features, convolution, hematoxylin and eosin.

\footnotetext{
${ }^{*}$ Corresponding author

Email address: dracoceanu@pucp.edu.pe (Daniel Racoceanu )

${ }^{1}$ School of Medical Science and Technology, Indian Institute of Technology, Kharagpur, West Bengal, India. ${ }^{+}$Joint Corresponding author.

${ }^{2}$ University Pierre and Marie Curie (UPMC - Paris 6), Paris France and with the Pontifical Catholic University from Peru (PUCP).
} 


\section{Introduction}

Breast cancer (BC) is increasing gradually in developed and developing countries (considered second most common cancer among women) [1, 2]. As per the Nottingham score, BC grading is done by the assessment of nuclear pleomorphism, tubule formation and mitotic count [3]. In the healthy breast, epithelial cell nuclei are uniform in size and shape. But in the case of malignancy, epithelial cell nuclei become non-uniform, darker and larger in shape. This transformation is called nuclear pleomorphism [4]. Tubule formation represents the percentage of malignant cells form regular duct structure. Mitotic count, one of the most important proliferation factors, carries significant diagnostic information required for $\mathrm{BC}$ histological grading. Indeed, it provides clues to estimate the aggressiveness and proliferation rate of the tumor [5] by assessing the severity of the disease. During mitotic cell division, the chromosome is divided into two identical chromosomes. The mitotic cell division process is characterised by four main phases: prophase, metaphase, anaphase and telophase [6]. Due to mutational changes in cell DNAs, there might be possibilities of uncontrolled cell division [7].

An individual observation area is usually called HPF [9]. Mitotic scoring is calculated based on the number of mitotic figures present in the high power field (HPF). If 0-11 mitotic figures are present in 10 neighbour HPFs, then the score will be 1 . If 12-22 mitotic figures are present in 10 neighbour HPFs, then the score will be 2, and the score will be 3 if more than 23 mitotic figures are visible in 10 neighbour HPFs [5, 8].

In histopathological image analysis, automatic mitosis detection exhibits various challenges. Firstly, the shape of mitotic figures is different in each of the phases. Secondly, in hematoxylin and eosin (H\&E) stained images, apart mitotic figures, some other similar objects like dense nuclei, lymphocytes, etc., are also present, inducing an important chance of misclassification. Therefore, due to some possible variation in the slide preparation (staining process, concentration, proportion, etc.), pathologists may miss some prominent mitotic figures. Moreover, manual detection of the mitotic figure is tedious and prone to intra-observer variability. Furthermore, in rural or urban areas with no/less expert pathologist, the manual assessment alone may provide wrong (or misleading) information. Automatic detection and count of the mitotic figure will not only attenuate these problems but also provide a safeguard to the doctors to consolidate their decision and support their commitment. In clinical practice, the pathologists assess the proliferated area present in the H\&E stained tissue slides under $40 \times$ magnification.

Literature shows that computer aided mitosis detection (see MITOS-ATYPIA14 [10] and MITOS 2012 [11, 12] challenges organised at ICPR) has not yet achieved enough performances (the maximum F-score gained was 0.734 in 2014) to envisage into routine implementation [13]. Therefore, an efficient and affordable deep learning model has been studied in this article. The proposed model is genuine and requires no prior knowledge about mitosis detection.

The key idea of the proposed architecture is based on two premises: (a) to 
learn deep learning features and handcrafted features from the ground truth annotated data and (b) classification between mitosis and non-mitosis. Small patches of size $71 \times 71$ have been used to train the model. The use of small patches in the proposed model localises the mitosis and non-mitosis in the images.

The paper is structured around VI sections: section I gives an introduction. The literature review is reported in Section II. Section III describes the dataset and proposed methodology. Results and discussion are discussed in Section IV and V. Finally, Section VI concludes and presents the perspectives of our work.

\section{LITERATURE REVIEW}

Many automatic machine learning techniques are already developed in mitosis detection using $H \& E$ images and a certain success has been witnessed. Due to imperfections in cutting and staining, various issues arise from the variability of tissue appearance. Most of the mitosis detection techniques are based on conventional imaging techniques. From the extensive literature survey, we can remark that there are very few studies dedicated to the deep learning approaches. Table 1 shows the characterisation of different mitosis detection methods using $\mathrm{BC}$ histological images. Table 2 illustrates comparative results of different BC dataset and strategies.

\subsection{Review Based on Conventional Techniques}

Table 1 shows maximum BC mitosis detection issues which have been resolved by color, texture and intensity related attributes. H. Irshad et al.,(2013) proposed a method where candidate cells were detected and segmented by extracting various channel features followed by Laplacian Gaussian, morphological operations and active contour [14]. They used ICPR 2012 dataset. Their proposed method achieved F-score $72 \%$ on Aperio and $63 \%$ on Hamamatsu scanner images. This study was further extended based on various textures, Hierarchical Model and $\mathrm{X}$ (HMAX) features [9]. This approach resulted in 0.76 recall, 0.75 precision and $0.76 \mathrm{~F}$-measure. The authors concluded that the result could be used for additional analysis. H. Irshad et al., (2014) proposed spatial characterisation and multispectral band selection technique for mitosis detection. Their algorithm achieved $67.35 \%$ detection rate and $63.74 \% \mathrm{~F}$-score [15]. The use of multispectral images using specifically designed spectral band selection algorithm for mitosis detection has been proposed in [16]. This algorithm achieved F-score 74\%, positive predictive value $76 \%$ and true positive rate $74 \%$.

In [17], a probabilistic model using phase-contrast microscopy images has been discussed. The algorithm has been designed in a two-step processes: (a) the detection of spatiotemporal patches and (b) localization of birth events. The results show that their approach outperforms the previous approaches concerning computational efficiency and detection accuracy. In the method proposed by [18], mitosis detection was done by extracting generic features and classifying by single and cascaded Adaboost classifier. The authors tested their model on ICPR 2012 contest dataset. The performance of the single classifier was $68 \%$ 
recall, $28.1 \%$ precision, $39.7 \%$ F-score, whereas, cascaded Adaboost classifier shows $54 \%$ recall, $62.7 \%$ precision, $58 \% \mathrm{~F}$-score. The authors suggested to use the ensemble of cascaded Adaboost classifier rather than single classifier along with the features which express better color variations and granular structure.

L. Roux et al., (2013) described the methods used for MITOS @ ICPR 2012 contest dataset. The participants to this first challenge in Digital Pathology used level set, active contour, thresholding, mathematical morphology along with radiometry, morphological features and texture features for the detection of mitosis [11]. The authors achieved F-measure 0.78 in mitosis detection. A. Liu et al., (2010) proposed the development of a mitosis event detection technique using hidden conditional random field. The model was designed into two parts. The candidate spatiotemporal sub-regions were segmented by image pre-conditioning and volumetric segmentation. This method achieved $85 \%$ recall and $95 \%$ precision [19]. M. schlachter et al.,(2010) used harmonic filters with rotation invariant to detect mitosis in colorectal cancer images. Their approach successfully detected the mitosis in colorectal cancer [20].

C.Y.Tao et al., (2007) proposed the use of a support vector machine classifier for mitotic figure detection using high-content screening data. The model tested on 99 cells and result shows $95.2 \%$ sensitivity and $95.8 \%$ specificity [21]. Another computerized mitosis detection technique has been described in [22]. The authors found strong agreement between pathological observation results and computerized technique. In [23], Exclusive Independent Component Analysis has been used for mitosis detection. The algorithm tested on ICPR 2012 dataset. They achieved an area under the curve $100 \%$. In [24], objective and texture based features have been used for mitosis detection. The acquired histology images firstly pre-processed by $2 \mathrm{D}$ anisotropic diffusion and morphological operations. Secondly, maximum likelihood estimation had been used for pixel-wise feature extraction. Finally, a SVM classifier has been used for classification. The test has been done on ICPR 2012 contest dataset images and achieved F-score of $70.11 \%$ for Hamamatsu images and $70.94 \%$ for Aperio XT scanner images. The work has been extended in [25]. R. Nateghi et al., (2014) presents an automatic mitosis detection method using cast function and Genetic optimization algorithm. The segmented mitosis and non-mitosis cells were classified using the SVM classifier. They achieved F-score $78.47 \%$ [26]. In [27], statistical detection algorithms, i.e., constrained energy minimization, matched filtering and adaptive coherence estimator has been used for mitosis detection. They used matched filter and achieved $80 \%$ accuracy.

In [28], the authors compared the manual and automatic mitosis detection algorithms. They suggested that there should be a sufficient number of the training dataset. A. S. Tripathi et al.,(2013) proposed a 2-Sieve model for mitosis detection. They have extracted Gray Level Entropy Matrix features and Multiresolution wavelet features on all the spectral bands. Classical data mining technique and dimensionality selection technique had been used in this model. Their results show positive predictive value $73.04 \%$ and sensitivity $82.35 \%$ [29]. The approach presented in [30], uses the whole slide histological images for mitotic cell extraction and visualization, using the multi-resolution graph-based 
technique. The accuracy of this unsupervised learning technique was improved by spatial refinement of label regularization.

In [31], the authors reviewed the graph-based methods for microscopic histopathology images. They addressed various methodologies and their corresponding time complexities. The authors concluded that graph based methods are capable of differencing tissue component based on neighborhood relationships and spatial arrangements. This tends to consume less time during training and testing phase. The authors of [32] proposed structure and function based diagnosis using tissue slides. They used histological images for measuring staining intensity in a gray level. Additionally, Shannon's and structural entropy and entropy flow had been derived from the mapped gray level datasets. The significant changes found in the entropy level suggested biological function and structure based diagnosis for rapid reporting and result.

\subsection{Review Based on Convolution and Deep Learning Techniques}

A deep neural network based mitotic figure detection technique was proposed for BC histology images [33]. This study was based on the deep max-pooling convolutional network. Their approach won the ICPR 2012 mitosis detection challenge by achieving an F-score of 0.782 . M.Veta et al., (2016) reported an assessment of object-level inter-observer variability on mitosis counting in BC [34]. The authors trained a deep learning model for mitosis counting followed by performance analysis based on an inter-observer agreement between automatic and manual method. Their framework consisted of four convolutional and two fully connected layers. The authors employed AMIDA13 dataset with image patch of size $63 \times 63$. They concluded that small objects create substantial disagreement so there should be size constraint in mitosis detection. The results of AMIDA13 challenge algorithms of 11 participants have been summarised in [35] and the outcomes of individual participants are discussed.

Table 1

Categorization of mitosis detection approaches

\begin{tabular}{|c|c|c|}
\hline \multicolumn{2}{|c|}{ Categorize } & Mitosis Detection Approaches \\
\hline \multirow{11}{*}{ Hand-Crafted features } & \multirow{4}{*}{ Texture based } & Cooccurrence features, scaleinvariant features, runlength features [19] \\
\hline & & Wavelet-based [36] \\
\hline & & Oriented gradient histograms $[\mathrm{HOG}][37]$ \\
\hline & & Generic features [17] \\
\hline & \multirow{6}{*}{ Color-based } & Blue ratio [19] \\
\hline & & Maximization of relative-entropy [11] \\
\hline & & Spectral band selection [38] \\
\hline & & Gamma-Gaussian Mixture Model [39] \\
\hline & & Chan-Vese level set [40] \\
\hline & & $\begin{array}{l}\text { SCILAIM software used for mitosis detection } \\
\text { using Feulgen Stained BC images [41] }\end{array}$ \\
\hline & Contextual learning based & Learning based [42] \\
\hline \multirow{5}{*}{\multicolumn{2}{|c|}{ Deep Learning }} & Convolutional neural network (CNN) [43] \\
\hline & & Deep CNN and max-pooling [10] \\
\hline & & CNN and Handcrafted Features [17] \\
\hline & & CNN and seeded blob features $[22]$ \\
\hline & & AggNet: Deep learning from crowd [44] \\
\hline
\end{tabular}

H.Wang et al., [47], [48] used CNN and handcrafted features for mitotic figure detection. The proposed model employs one convolution, one pooling 


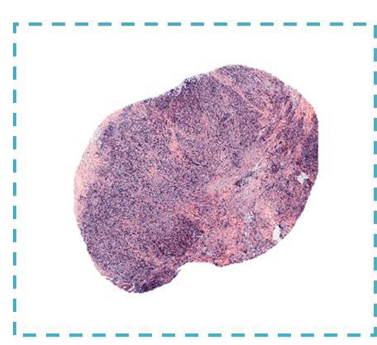

Whole Slide Image

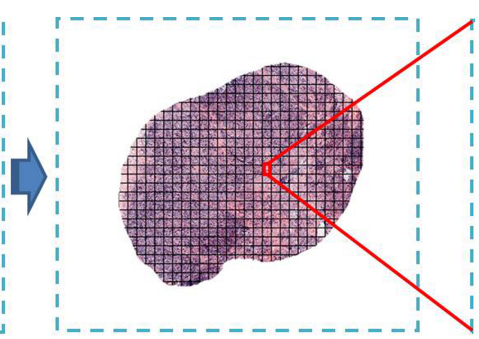

Whole slide image with grid

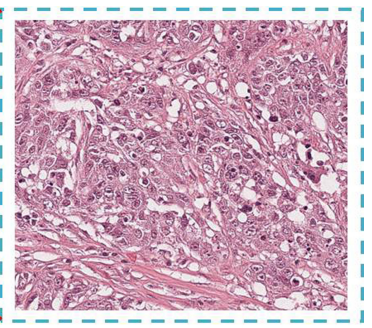

HPF image (40X magnification)

Figure 1: High power field image (40× magnification) generation

Table 2

Comparative results of different dataset and methods

\begin{tabular}{|c|c|c|c|c|c|}
\hline Reference & Dataset Used & F-score & Precision & Recall & Accuracy \\
\hline [45] & MITOS-ATYPIA-14 & 0.734 & 0.826 & 0.66 & - \\
\hline [10] & \multirow{17}{*}{$\begin{array}{c}\text { ICPR } 2012 \text { - Mitosis } \\
\text { detection contest }\end{array}$} & 0.782 & 0.88 & 0.70 & - \\
\hline [15] & & $\begin{array}{c}0.70 \text { (Aperio images) } \\
0.56 \text { (Hamamatsu images) }\end{array}$ & $\begin{array}{c}0.74 \text { (Aperio images), } \\
0.71 \text { (Hamamatsu images) }\end{array}$ & & - \\
\hline [22] & & 0.659 & 0.74 & 0.59 & - \\
\hline [39] & & 0.513 & 0.46 & 0.57 & - \\
\hline [17] & & 0.397 & 0.28 & 0.68 & - \\
\hline$[42]$ & & 0.374 & 0.14 & 0.80 & - \\
\hline [14] & & 0.7345 & 0.84 & 0.65 & - \\
\hline [19] & & 0.76 & 0.75 & 0.76 & - \\
\hline [20] & & 0.34 & - & - & - \\
\hline [21] & & 0.64 & - & 0.67 & - \\
\hline [26] & & - & - & - & 0.84 \\
\hline$[27],[29]$ & & $\begin{array}{c}0.7094 \text { (Aperio images), } \\
0.7011 \text { (Hamamatsu images) }\end{array}$ & - & - & - \\
\hline$[16$ ] & & 0.7847 & 0.80 & 0.77 & - \\
\hline [30] & & 0.74 & 0.76 & - & - \\
\hline [33] & & - & - & - & 0.859 \\
\hline [30] & & - & 0.7304 & 0.8235 & - \\
\hline [36] & & - & - & 0.8680 & 0.8794 \\
\hline [14] & AMIDA13 & 0.319 & - & - & - \\
\hline [46] & & 0.6133 & - & - & 0.8695 \\
\hline
\end{tabular}

and one fully-connected layer. The convolution layer contains 64 neurons; the pooling layer comprises of 128 neurons and the fully-connected layer contains 256 neurons. The parameters of the each layer were fixed, i.e. $8 \times 8$ convolution kernel size, $2 \times 2$ pooling kernel size. The handcrafted features used in the model are morphological (eccentricity, area, compactness, smoothness, etc.), textural (concurrence features and run-length features) and intensity features (median, mean, variance, interquartile range, maximum/minimum ratio, range etc.). They tested the model on ICPR 2012 dataset and achieved F-measure of 0.7345. Another CNN based mitosis detection technique has been described in [49]. They used CNN based feature along with nuclear features in this model. They tested on the ICPR 2012 dataset and achieved F-score 0.589 on the multispectral scanner and F-score 0.659 on the color scanner. They concluded that this technique provides majority mitosis with fair precision value $(0.747$ in Aperio, 0.759 in Hamamatsu and 0.738 in the multispectral scanner). In [44], convolutional neural network along with additional aggregation layer for 
learning from crowds have been employed for mitosis detection. The methodology used AMIDA13 Challenge BC histopathology image dataset achieved an overall F1-score of 0.433 , a precision of 0.441 and a recall of 0.424 .

Unlike most of the deep learning models involving convolutional, resizing and handcrafted layers, the approach presented in this paper employs deep learning for high-level feature learning. In our proposed deep architecture, the size of mitosis as well as non-mitosis patches were assigned the pixel size of $71 \times 71$ at $40 \times$ magnification. Every patch contains only a single object, i.e. mitosis or non-mitosis, that would be suitable for the building of a supervised deep learning model. Therefore, we included handcrafted features instead of only deep learning network (DLN). On the other hand, to extract some additional features, handcrafted features are trained in a supervised fashion. Moreover, this supervised technique is much more efficient than the usual deep learning techniques. This method permits us to detect mitosis and non-mitosis efficiently from the large cohort of $\mathrm{BC}$ histological images.

The novelties of this paper are:

- The proposed deep architecture is efficient to learn and classify from a large number of non-annotated histological image data.

- The handcrafted features have been merged with the DLN features and all the features directly fed to the first fully connected layer which represents high-level representation of mitosis and non-mitosis.

- Handcrafted layer allows quick traversal through large images for detection of single mitosis more accurately and efficiently.

To summarise, this paper incorporates the handcrafted features and deep learning based high-level features for mitosis detection. Our proposed approach is efficiently detecting mitosis patches from whole slide HPF images.

\section{EXPERIMENTAL SETUP}

\subsection{Dataset}

The proposed method has been assessed on the MITOS-ATYPIA-14 (1,136 training and 991 testing frames), ICPR-2012 (35 training and 15 testing frames) and AMIDA-13 (326 training and 259 testing frames) dataset [10, 12, 50]. The slides were stained with routine $\mathrm{H} \& \mathrm{E}$ stain. The images were scanned by Hamamatsu NanoZoomer 2.0-HT and Aperio Scanscope XT. The size of MITOSATYPIA-14 images were for Aperio scanner $1539 \times 1376$ pixels $($ width $\times$ height $)$, 1 pixel $=0.2456 \mu \mathrm{m}$ and Hamamatsu scanner $1663 \times 1485$ pixels $($ width $\times$ height $)$, 1 pixel $=0.2273 \mu \mathrm{m}$. The size of ICPR-2012 images were for Aperio $2048 \times 2048$ pixels (width $\times$ height), 1 pixel $=0.2456 \mu \mathrm{m}$ and Hamamatsu scanner $2252 \times 2250$ pixels (width $\times$ height), 1 pixel $=0.2273 \mu \mathrm{m}$. The size of AMIDA-13 images were for Aperio scanner $2000 \times 2000$ pixels $($ width $\times$ height $), 1$ pixel $=0.2456 \mu \mathrm{m}$. For all the dataset image, dimension is different, but magnification $(40 \times)$ and pixel resolution are fixed, for a particular type of scanner. 


\subsection{Deep Learning}

\subsubsection{Convolutional Network (CN)}

It is an arrangement of an order of hidden layers. Let's, CN denoted by ' $f$ ' and order of ' $l$ ' layers / functions $\left(f_{1}, f_{2}, f_{3}, \ldots \ldots \ldots f_{l}\right)$ that feed to the input ' $w$ ' vector to an output ' $x$ ' vector. Mathematically, we can write [39] [51] [52]:

$$
\begin{aligned}
x & =f\left(w ; v_{1}, v_{2}, v_{3} \ldots . . v_{l}\right) \\
& =f_{1}\left(. ; w_{1}\right) \circ f_{2}\left(. ; w_{2}\right) \circ \ldots f_{l-1}\left(. ; w_{l-1}\right) \circ f_{l}\left(. ; w_{l}\right)
\end{aligned}
$$

Here, ' $v_{l}$ ' represents weight and bias vector for $l^{\text {th }}$ layer, i.e., ' $f_{l}$ '. ' $f_{l}$ ' has been used to perform pooling, non-linear activation and convolution with bank of filters. As per stochastic gradient descent method, numerical optimisation problem is calculated by:

$$
O p t_{v_{1}, v_{2} \ldots v_{l}}=\frac{1}{M} \sum_{i=1}^{M} \Omega\left(f\left(w^{i} ; v_{1}, v_{2}, v_{3} \ldots v_{l}\right), x^{i}\right)
$$

Where ' $\Omega$ ' denotes loss function, ' $M$ ' is number of data.

\subsubsection{Rectified Linear Unit}

Rectified linear unit (ReLU) works as an activation function. In caffe, ReLU with gradient descent is defined as [42]:

$$
g(r)=\max (0, r)
$$

Where, ' $g$ ' denotes model's output function with an input ' $r$ '.

\subsubsection{Pooling Layer}

Pooling layer is employed to down-samples the image size by a constant factor, i.e., 2 or 3 [37]. This layer improves the filter selectivity. This layer produces single output by sub-sampling the convolutional layer. We have used max-pooling layers, which consider a maximum of the block, in the model.

\subsubsection{Fully Connected Layer}

In deep learning, fully connected (FC) layer works as a classifier. FC layer is not spatially located and serves as a simple vector. This layer produces an output like single vector. In the proposed model, the fully connected layer height and width of each blob are set to 1 .

\subsubsection{Dropout Layer}

Dropout layer is basically used to set the hidden neurone output to zero [42]. In the proposed model, dropout value is set to 0.05 . The dropout neurones will not be useful for back-propagation or forward pass. This layer reduces the co-adaption complexity of neurones. In the proposed architecture, in order to avoid overfitting, the dropout layer has been included after the first fully connected layer. 


\subsubsection{Handcrafted Features Layer}

A lot of research articles has been dedicated to select handcrafted features in conventional mitosis detection techniques. However, it is tedious and timeconsuming to mathematically describe the domain specific knowledge and human intuition. Moreover, human heuristics may not guarantee the performance of a feature on a related machine learning problem. An increasing number of papers demonstrate that handcrafted features and deep learning alone provide the best result in computer vision problem. Inspired by this concept, we compile both the techniques to achieve a better result. The handcrafted features played a vital role in the proposed architecture. We used handcrafted features issued from previous medical challenges MITOS @ ICPR 2012, AMIDA-13, and projects (MICO ANR TecSan) expertise. In our methodology, handcrafted features mainly consist of morphological, textural and intensity features $[53,54,55]$. The morphological features mainly signify characteristics of mitosis shape. The texture and intensity features represent textural and statistical characteristics of mitosis region. All the features have been extracted from the masked RGB image patches of training dataset. The table 3 shows the 55 Hand-crafted features extracted from the mitosis and non-mitosis image patches (* represents significant).

\subsection{The Proposed Deep Learning Architecture}

At first, whole slide images were processed using openslide software to get HPF images. Fig. 1 shows the flow of HPF image generation (40× magnification), an image adopted from [56]. Then, the HPF colour images have been converted into blue ratio images [Fig. 2]. The main goal of the conversion is to find the most prominent and high brightness objects within the image. Mathematically, the conversion from RGB to Blue Ratio image is denoted by the following equation [46]:

$$
\text { BlueRatio }=\frac{100 \times B}{1+R+G} \times \frac{256}{1+R+G+B}
$$

Here, ' $R$ ' denotes red channel intensity ' $B$ ' denotes blue channel intensity and ' $G$ ' denotes green channel intensity.

The blue ratio images are then processed by morphological erosion and dilation operations. The gray scale erosion is denoted by the below equation [57]:

$$
(r \ominus s)(p, q)=\min \left\{r\left(p+p \prime, q+q^{\prime}\right)-s\left(p \prime, q^{\prime}\right) \mid\left(p^{\prime}, q^{\prime}\right) \in V_{s}\right\}
$$

Here, (r $\ominus \mathrm{s})$ represents binary erosion of $r$ and the structuring element $s . V_{s}$ represents the domain of the structuring element $s$ and $r(p, q)$ assigned to $+\infty$. Now, for flat structuring element, gray scale erosion will be,

$$
(r \ominus s)(p, q)=\min \left\{r(p+p \prime, q+q \prime) \mid(p \prime, q \prime) \in V_{s}\right\}
$$


Table 3 The 55 Hand-crafted features extracted from the mitosis and non-mitosis image patches

\begin{tabular}{|c|c|c|c|}
\hline Sl. No. & Features & Mitosis & Non-mitosis \\
\hline 1 & Area* & $7.475 \pm 1.769$ & $9.022 \pm 2.246$ \\
\hline 2 & Perimeter* & $27.595 \pm 6.936$ & $31.824 \pm 6.304$ \\
\hline 3 & Compactness & $112.158 \pm 22.610$ & $113.890 \pm 23.592$ \\
\hline 4 & Eccentricity & $0.841 \pm 0.189$ & $0.824 \pm 0.210$ \\
\hline 5 & Zernike moment & $\mathrm{NaN}$ & $\mathrm{NaN}$ \\
\hline 6 & Area equivalent diameter & $8.973 \pm 0.949$ & $8.974 \pm 1.134$ \\
\hline 7 & Perimeter equivalent diameter* & $16.919 \pm 4.005$ & $20.4224 \pm 5.084$ \\
\hline 8 & Form factor* & $0.334 \pm 0.042$ & $0.115 \pm 0.024$ \\
\hline 9 & Convex area* & $66.694 \pm 16.807$ & $79.319 \pm 17.696$ \\
\hline 10 & Solidity* & $0.852 \pm 0.050$ & $0.405 \pm 0.059$ \\
\hline 11 & Roundness* & $0.658 \pm 0.123$ & $0.375 \pm 0.114$ \\
\hline 12 & Aspect Ratio* & $1.395 \pm 0.254$ & $1.057 \pm 0.273$ \\
\hline 13 & Concavity* & $11.540 \pm 5.669$ & $15.160 \pm 4.733$ \\
\hline 14 & Rectangularity & $0.698 \pm 0.040$ & $0.682 \pm 0.059$ \\
\hline 15 & Fourier descriptor* & $62007.048 \pm 16264.126$ & $70987.962 \pm 12160.941$ \\
\hline 16 & Mean intensity & $1.844 \pm 0.293$ & $1.798 \pm 0.358$ \\
\hline 17 & Energy & $1.825 \pm 0.291$ & $1.842 \pm 0.354$ \\
\hline 18 & Homogeneity & $2.237 \pm 0.292$ & $2.267 \pm 0.354$ \\
\hline 19 & Spot area ratio & $2.827 \pm 0.289$ & $2.787 \pm 0.384$ \\
\hline 20 & Contour,irregularity & $0.165 \pm 0.223$ & $0.242 \pm 0.356$ \\
\hline 21 & Area irregularity & $0.223 \pm 0.068$ & $0.218 \pm 0.067$ \\
\hline 22 & Area overlapped,Ratio & $0.177 \pm 0.056$ & $0.179 \pm 0.089$ \\
\hline 23 & Euler number & $0.218 \pm 0.067$ & $0.223 \pm 0.066$ \\
\hline 24 & Extent & $0.775 \pm 0.041$ & $0.281 \pm 0.081$ \\
\hline 25 & Major axis length & $0.796 \pm 0.052$ & $0.800 \pm 0.044$ \\
\hline 26 & Minor axis length & $0.757 \pm 0.043$ & $0.754 \pm 0.061$ \\
\hline 27 & Average radial ratio & $0.704 \pm 0.048$ & $0.705 \pm 0.051$ \\
\hline 28 & Hausdorff dimension* & $15.168 \pm 0.389$ & $10.743 \pm 0.056$ \\
\hline 29 & Smoothness* & $13.326 \pm 7.901$ & $26.354 \pm 9.227$ \\
\hline 30 & Standard distance,ratio* & $14.540 \pm 8.038$ & $23.871 \pm 8.004$ \\
\hline 31 & Autocorrelation* & $13.460 \pm 7.623$ & $26.104 \pm 8.834$ \\
\hline 32 & Contrast* & $33.699 \pm 1.460$ & $24.098 \pm 8.166$ \\
\hline 33 & Correlation-1* & $3.370 \pm 1.342$ & $6.602 \pm 1.249$ \\
\hline 34 & Correlation-2* & $3.605 \pm 1.361$ & $6.203 \pm 1.174$ \\
\hline 35 & Cluster Prominence & $3.395 \pm 1.290$ & $3.576 \pm 1.229$ \\
\hline 36 & Cluster Shade & $0.095 \pm 0.028$ & $0.105 \pm 0.034$ \\
\hline 37 & Dissimilarity & $0.125 \pm 0.035$ & $0.114 \pm 0.044$ \\
\hline 38 & Skewness * & $0.502 \pm 0.029$ & $0.927 \pm 0.033$ \\
\hline 39 & Entropy* & $0.322 \pm 0.034$ & $0.814 \pm 0.039$ \\
\hline 40 & Homogeneity & $0.600 \pm 0.077$ & $0.605 \pm 0.095$ \\
\hline 41 & Maximum probability & $0.675 \pm 0.079$ & $0.662 \pm 0.097$ \\
\hline 42 & Sum of squares & $0.617 \pm 0.077$ & $0.603 \pm 0.092$ \\
\hline 43 & Sum average & $0.676 \pm 0.078$ & $0.692 \pm 0.096$ \\
\hline 44 & Sum variance* & $0.798 \pm 0.178$ & $1.008 \pm 0.209$ \\
\hline 45 & Sum entropy & $0.948 \pm 0.178$ & $0.957 \pm 0.219$ \\
\hline 46 & Difference variance & $0.978 \pm 0.168$ & $0.963 \pm 0.200$ \\
\hline 47 & Difference entropy & $0.054 \pm 0.176$ & $0.057 \pm 0.212$ \\
\hline 48 & Information measure of correlation-1 & $0.962 \pm 0.010$ & $0.958 \pm 0.012$ \\
\hline 49 & Information measure of correlation- 2 & $0.952 \pm 0.013$ & $0.949 \pm 0.016$ \\
\hline 50 & Inverse difference homogeneity & $0.960 \pm 0.011$ & $0.955 \pm 0.012$ \\
\hline 51 & Inverse difference normalized & $0.943 \pm 0.013$ & $0.941 \pm 0.014$ \\
\hline 52 & Inverse difference moment normalized* & $0.824 \pm 0.052$ & $0.467 \pm 0.067$ \\
\hline 53 & Maximum ratio* & $0.815 \pm 0.053$ & $0.359 \pm 0.069$ \\
\hline 54 & Minimum ratio* & $0.822 \pm 0.051$ & $0.566 \pm 0.065$ \\
\hline 55 & Haralick feature* & $0.816 \pm 0.053$ & $0.459 \pm 0.068$ \\
\hline
\end{tabular}




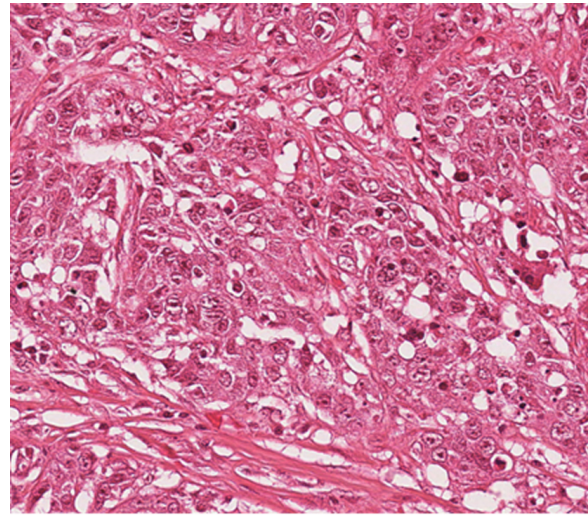

(a)

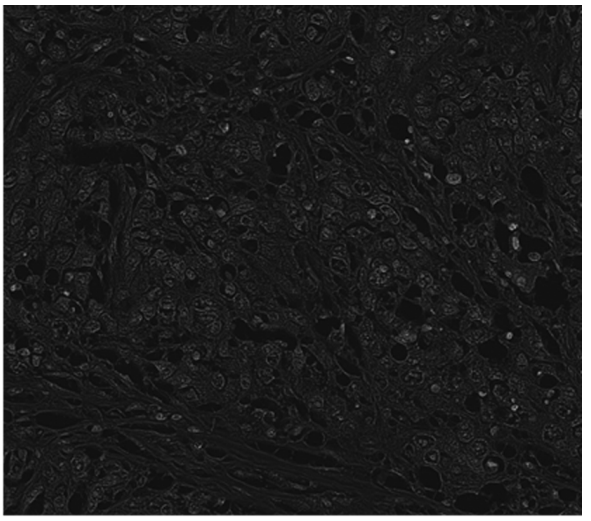

(b)

Figure 2: RGB to Blue ratio image conversion (a) original RGB image; (b) blue ratio image

Where, $s(p, q)=0$.

In the same way, the dilation equation for flat structuring element gray scale image is,

$$
(r \oplus s)(p, q)=\max \left\{r\left(p-p \prime, q-q^{\prime}\right) \mid(p \prime, q \prime) \in V_{s}\right\}
$$

Erosion and dilation help in removing unnecessary objects in a blue ratio image. We found the centroid of each objects. On the basis of centroid, we crop the image into small patches (mitosis and non-mitosis). The small patches of $w \times w=71 \times 71=5041$ pixels are collected and passed to the model. To contain mitosis/non-mitosis within the above mentioned patch size under $40 \times$ magnification resolution image is enough. The non-mitosis patches do not contain any mitosis or any part of mitosis and vice-versa. For the proposed architecture, we rotated patches into $0^{\circ}, 45^{\circ}, 90^{\circ}$ and $180^{\circ}$ to diminish the rotation-variant issue of the input features and to increase the number of training dataset. All patches are in RGB image format. Let's, the number of colour channels is denoted by $(\mathrm{T})=3$. In the input layer of the proposed deep learning architecture, patch represents vector pixel intensity in the form of column vector. Therefore, there are $d_{x}=s_{0}=3 \times 71 \times 71=15123$ inputs. Here, $2 \times 2$ region corresponds to 4 input pixels. This region is called Local receptive fields (LRF). Now, we move the LRF across the whole image. In the first input layer the LRF is being moved by one layer pixel at a time. This length is called stride length.

The proposed deep learning model has been developed using CAFFE deep learning framework [38]. The model contains mainly two sections, i.e., the first section is deep architecture and the second section is handcrafted features. The deep architecture contains five convolution layer, four max-pooling layer, four ReLU, and two fully connected layer. ReLU has been used after each convolutional layer [36]. Dropout layer has been included after first fully connected layer [43]. For better result, the dropout ratio is set to 0.05 . Table 4 shows 
architectural details of the proposed deep framework. The workflow diagram of the proposed deep framework has been shown in Fig. 3. The Fig. 4 illustrates a flow diagram of the handcrafted feature generation. The overall methodology has been described in Fig. 5. This model is inspired by [42] and [40]. Our proposed model learns from the labeled data.

Table 4

Proposed deep learning architecture

\begin{tabular}{|c|c|c|c|c|}
\hline Layer & Type & Maps & Neurons & Filter size \\
\hline 0 & Input image & 3 & $71 \times 71$ & - \\
\hline 1 & Conv & 90 & $70 \times 70$ & $2 \times 2$ \\
\hline 2 & Max-pooling & 90 & $35 \times 35$ & $2 \times 2$ \\
\hline 3 & Conv & 256 & $32 \times 32$ & $4 \times 4$ \\
\hline 4 & Max-pooling & 256 & $16 \times 16$ & $2 \times 2$ \\
\hline 5 & Conv & 384 & $14 \times 14$ & $3 \times 3$ \\
\hline 6 & Max-pooling & 384 & $7 \times 7$ & $2 \times 2$ \\
\hline 7 & Conv & 512 & $6 \times 6$ & $2 \times 2$ \\
\hline 8 & Max-pooling & 512 & $3 \times 3$ & $2 \times 2$ \\
\hline 9 & Conv & 512 & $2 \times 2$ & $2 \times 2$ \\
\hline 10 & FC-1 & - & 1000 & $1 \times 1$ \\
\hline 11 & FC-2 & - & 2 & $1 \times 1$ \\
\hline
\end{tabular}

\subsection{Parameter Initialisation and Training}

Before training, we initialise few relevant parameters (e.g., training batch size, validation batch size, the number of testing iteration, etc.). We have total $11,921(6,800$ mitosis $+5,121$ non-mitosis $)$ training image patches $\left(t_{s}\right)$ and 4323 validation image patches $\left(v_{s}\right)$. Training batch size is calculated by the total number of training images processed together in a single batch. In our proposed model training batch size $\left(t_{b s}\right)$ is set to 256. Alternatively, validation batch size $\left(v_{b s}\right)$ is the total number of validation images processed together for test phase. In the proposed model, ' $v_{b s}$ ' is set to 50 . Testing iteration $\left(\right.$ test $\left._{i t r}\right)$ is calculated as: test $t_{i t r}=\left(v_{s} / v_{b s}\right)$. Testing interval $\left(\right.$ test $\left._{i n v}\right)$ determines how often you validate the model. Here 'test ${ }_{i n v}$ ' is set to 5000. So to cover the entire training set or completing an epoch, we have to run $\left(t_{s} / t_{b s}\right)$ iterations. Maximum iteration is set to 300,000 , weight decay 0.005 , learning rate 0.01 and momentum 0.9. The architecture was trained using stochastic gradient descent approach.

\section{EXPERIMENTAL RESULTS}

\subsection{Qualitative Results}

Total 55 features among all categories have been extracted. After that, the student's t-test has been performed and we got 24 significant ( $p<0.05,95 \%$ confidence interval) features out of 55 features. Fig. 6 shows the correlation matrix 


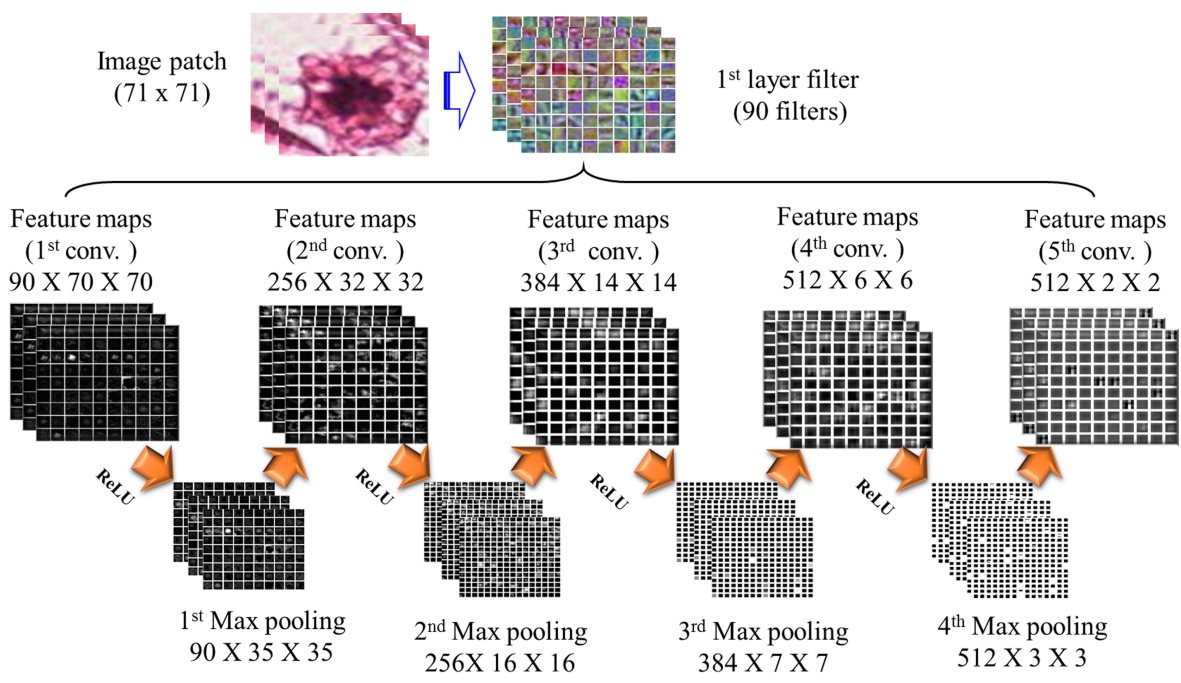

Figure 3: Flow diagram of the proposed deep learning network

Table 5

Performance measures for mitosis detection

\begin{tabular}{|c|c|c|c|c|}
\hline Scanner types & Confusion Matrix & Precision & Recall & F-score \\
\hline A perio & $172 \quad 23$ & \multirow{2}{*}{0.92} & \multirow{2}{*}{0.88} & \multirow{2}{*}{0.90} \\
\hline & $13 \quad 160$ & & & \\
\hline Hamamatsu & $\begin{array}{lr}171 & 24 \\
13 & 160\end{array}$ & 0.92 & 0.88 & 0.90 \\
\hline \multicolumn{2}{|r|}{ rage } & 0.92 & 0.88 & 0.90 \\
\hline
\end{tabular}

for 24 global features $(\mathrm{p}<0.05)$ for image classification. $|\operatorname{Corrcoe} f(x, y)|=1$ means highest correlation and $|\operatorname{Corrcoef}(x, y)|=0$ indicates no correlation. The visualisation of first layer filters and trained high-level (i.e., first, second, third, fourth and fifth hidden layer) features are shown in the Fig. 7. Only 100 feature maps have been shown in each hidden layer to visualise the maps clearly. These features illustrate the proposed deep architecture, allowing the detection of mitosis from the image patches.

The qualitative and detection results of the proposed architecture have been shown in Fig. 8 and Fig. 9 respectively. In these images, the yellow circle represents the true mitosis; the green circle represents the non-mitosis. These results suggested that the proposed architecture is working well for better detection of mitosis and non-mitosis.

\subsection{Quantitative Evaluation}

According to the MITOS-ATYPIA-14 challenge evaluation criteria, if the coordinates of the detected mitosis centroid to the ground truth mitosis centroid 


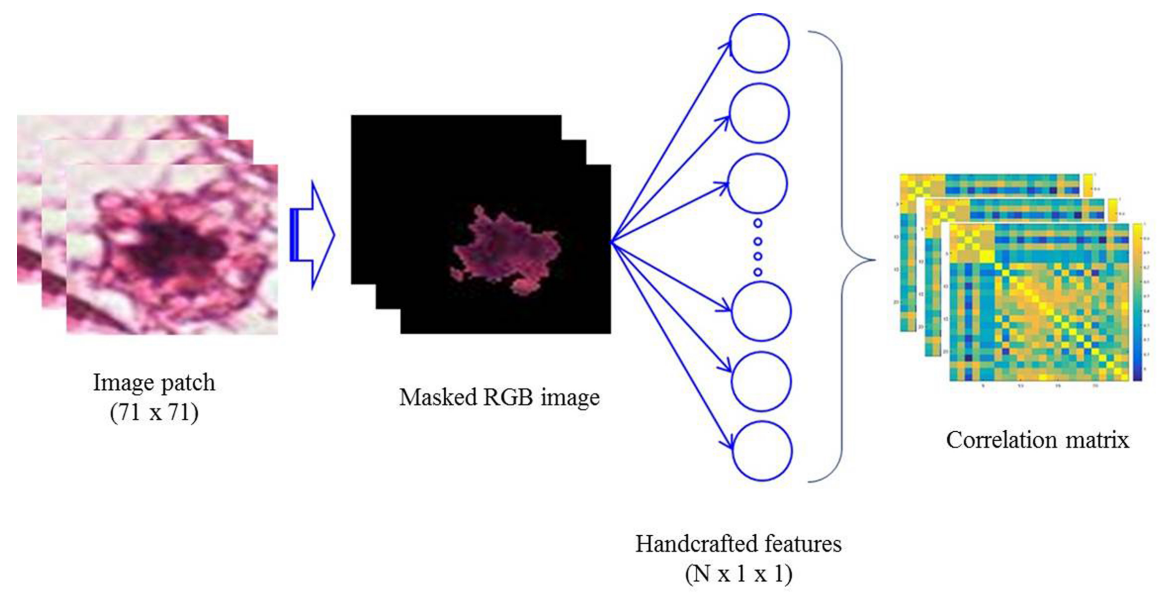

Figure 4: Work flow diagram of the handcrafted features generation

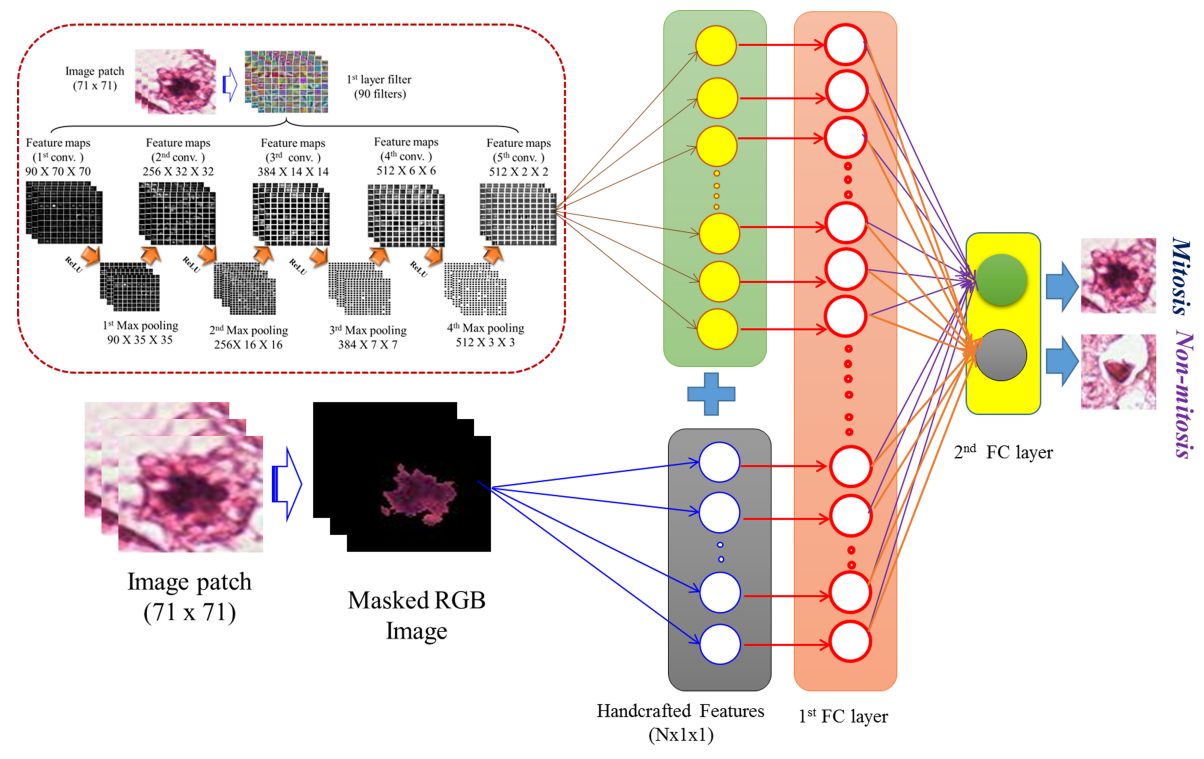

Figure 5: Flow diagram of the proposed methodology 


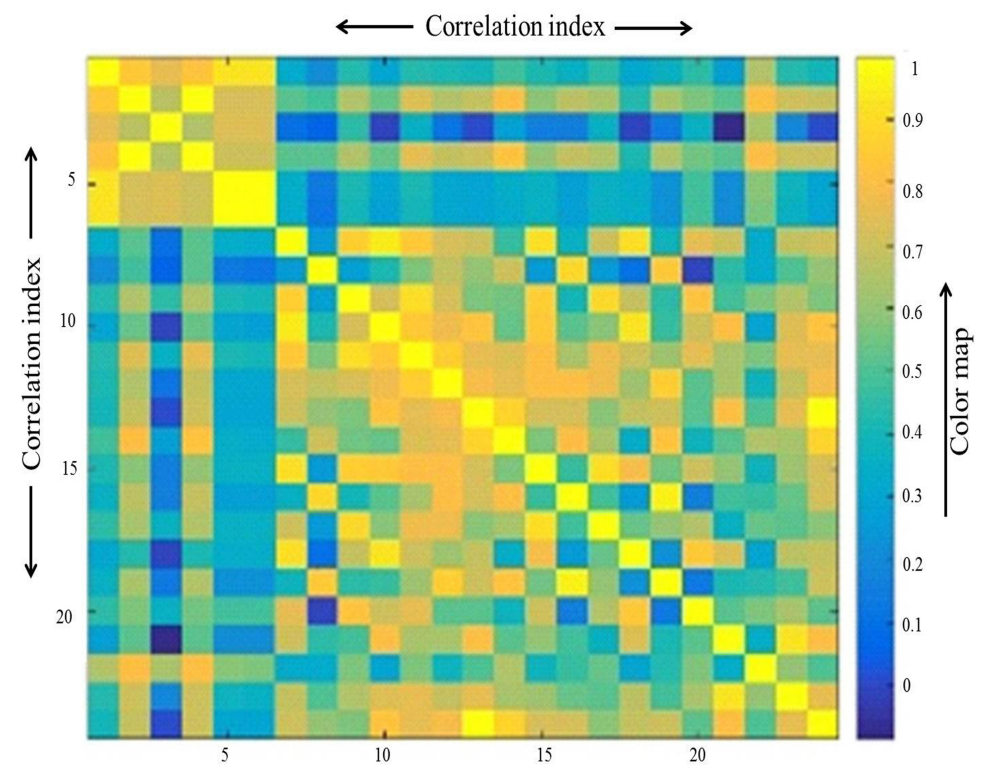

Figure 6: Correlation matrix for 24 global features for image classification

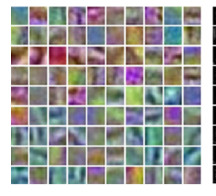

(a)

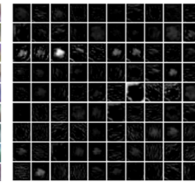

(b)

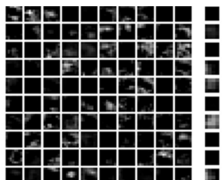

(c)

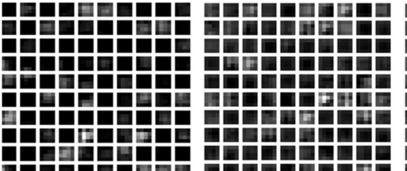

(e)

Figure 7: Shows filters and learned features maps of the proposed deep network, (a) first layer filter (b) first hidden layer $90(10 \times 9)$ units. (c) second hidden layer $100(10 \times 10)$ units. (d) third hidden layer $100(10 \times 10)$ units. $(e)$ fourth hidden layer $100(10 \times 10)$ units. (f) fifth hidden layer $100(10 \times 10)$

Table 6

5 -fold cross-validation

\begin{tabular}{|c|c|c|c|}
\hline Cross-Validation & $\boldsymbol{P r}$ & $\boldsymbol{R e}$ & $\boldsymbol{F}$-score \\
\hline 1 st FOLD & 0.92 & 0.88 & 0.90 \\
\hline 2nd FOLD & 0.92 & 0.90 & 0.90 \\
\hline 3rd FOLD & 0.91 & 0.88 & 0.89 \\
\hline 4th FOLD & 0.92 & 0.88 & 0.90 \\
\hline 5th FOLD & 0.92 & 0.87 & 0.89 \\
\hline Average & 0.92 & 0.88 & 0.90 \\
\hline
\end{tabular}




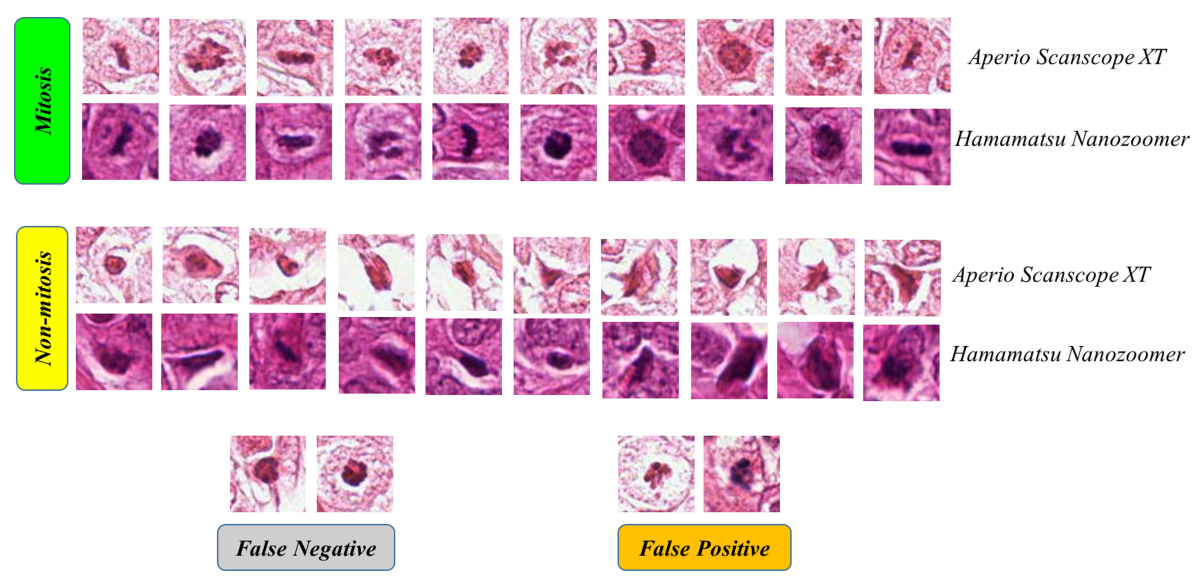

Figure 8: The mitosis detection results

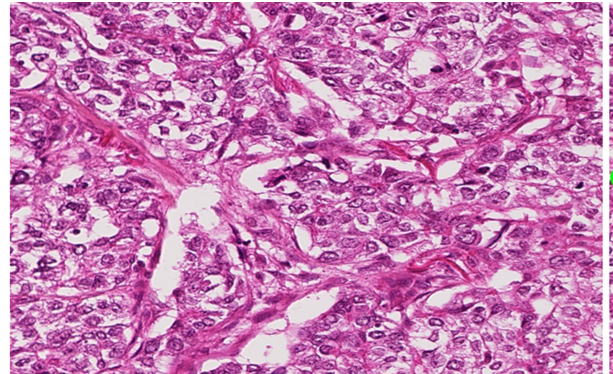

(a)

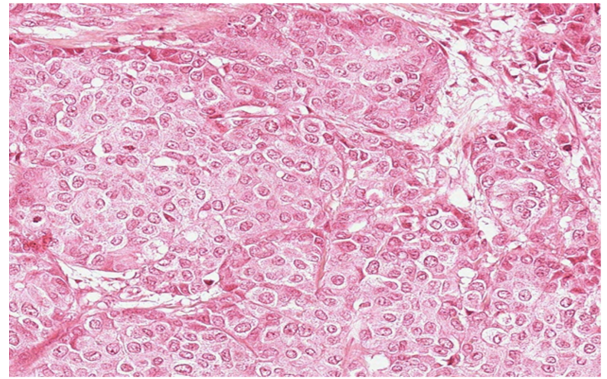

(c)

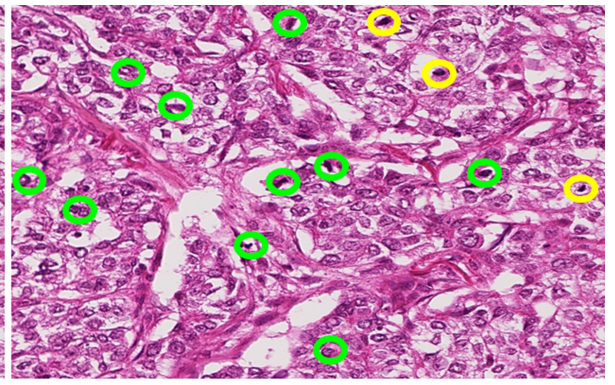

(b)

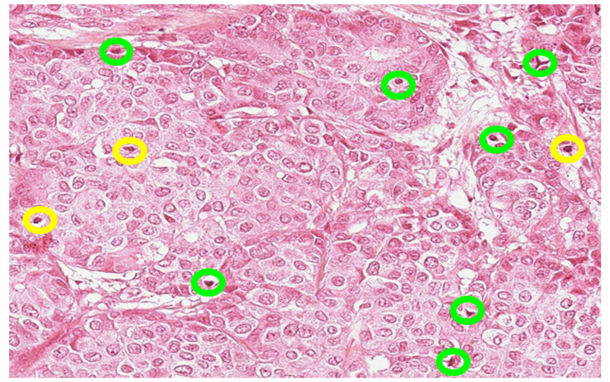

(d)

Figure 9: The mitosis detection results on HPF images at $40 \times$ magnification, Hamamatsu scanner: (a) original image, (b) final result mitosis (yellow circle) and non-mitosis (green circle); Aperio scanner: (c) original image, (d) final result mitosis (yellow circle) and non-mitosis (green circle) 


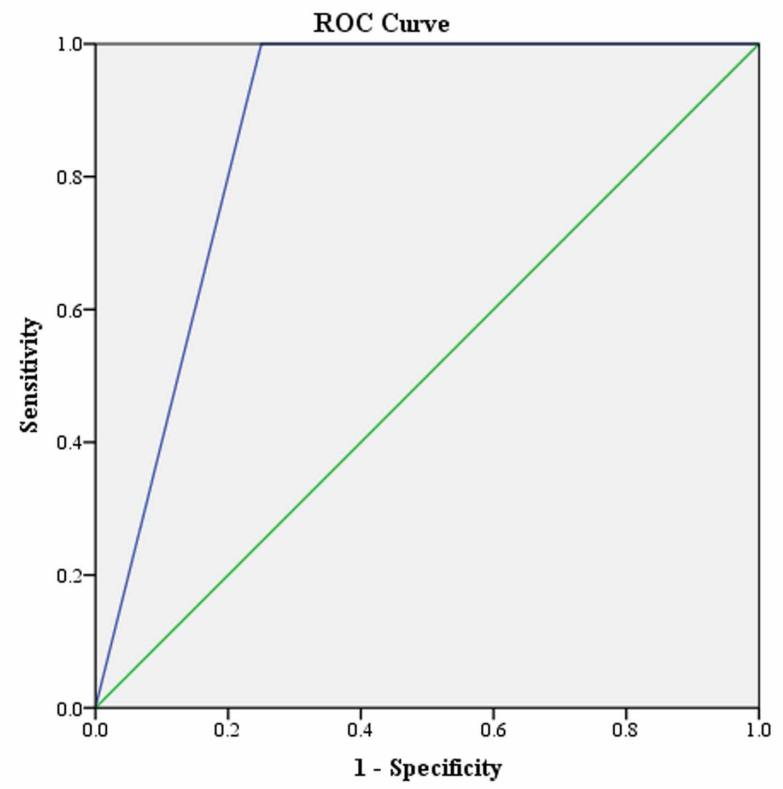

Figure 10: ROC curve

Table 7

Comparison with other methodologies

\begin{tabular}{|c|c|c|c|}
\hline References & $\boldsymbol{P r}$ & $\boldsymbol{R e}$ & $\boldsymbol{F}$-score \\
\hline$[13]$ & 0.83 & 0.66 & - \\
\hline$[47]$ & 0.84 & 0.65 & 0.73 \\
\hline$[33]$ & 0.88 & 0.70 & 0.78 \\
\hline Proposed method & 0.92 & 0.88 & 0.90 \\
\hline
\end{tabular}

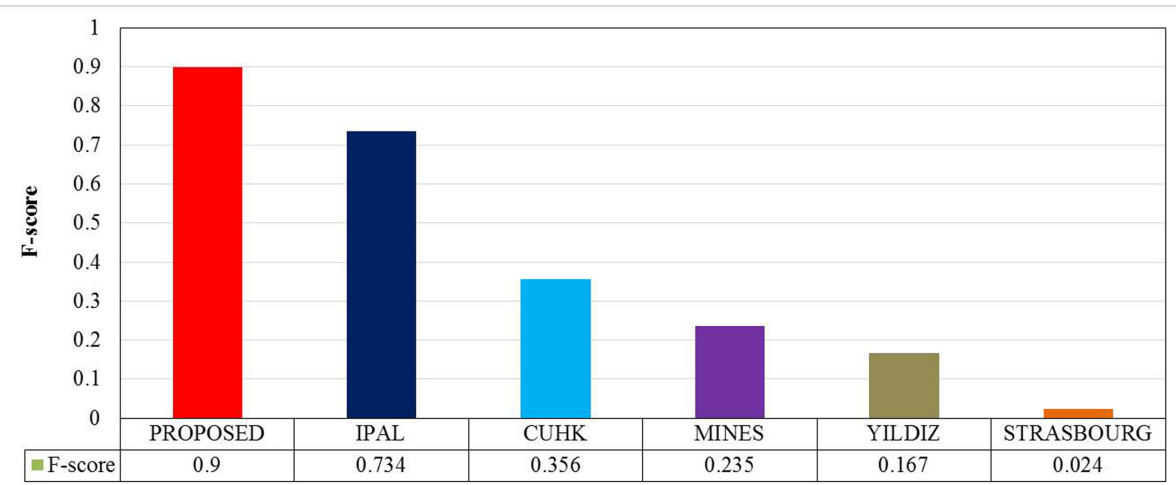

Figure 11: Comparison of F-score values using various methods on MITOS-ATYPIA-14 dataset 
Table 8

Performance with/without handcrafted features

\begin{tabular}{|c|c|c|c|}
\hline Proposed Method & $\boldsymbol{P r}$ & $\boldsymbol{R e}$ & $\boldsymbol{F}$-score \\
\hline Without handcrafted features & 0.86 & 0.69 & 0.76 \\
\hline With handcrafted features & 0.92 & 0.88 & 0.90 \\
\hline
\end{tabular}

Table 9

Performance based on various combinations of training and testing dataset

\begin{tabular}{|c|c|c|c|c|}
\hline Training images (\%) & Testing images (\%) & $\boldsymbol{P r}$ & $\boldsymbol{R e}$ & $\boldsymbol{F}$-score \\
\hline 25 & 75 & 0.92 & 0.88 & 0.90 \\
\hline 50 & 50 & 0.93 & 0.88 & 0.90 \\
\hline 75 & 25 & 0.94 & 0.89 & 0.91 \\
\hline
\end{tabular}

are within a range of $8 \mu \mathrm{m}$, then the detected mitosis will be considered as true positive [10]. The model is quantitatively evaluated using precision $(\mathrm{Pr})$, recall(Re) and F-Score [58].

$$
\begin{gathered}
\text { Fscore }=2 \times \frac{\operatorname{Pr} \times R e}{\operatorname{Pr}+R e} \\
\operatorname{Pr}=\frac{\text { True Positive }}{\text { True Positive }+ \text { False Positive }} \\
\operatorname{Re}=\frac{\text { True Positive }}{\text { True Positive }+ \text { False Positive }}
\end{gathered}
$$

Table 5 shows the performance measures of Hamamatsu NanoZoomer 2.0-HT and Aperio Scanscope XT scanner images. The proposed model achieved 0.92 precision, 0.88 recall and $0.90 \mathrm{~F}$-score value. The ROC curve is shown in Fig. 10. The area under the curve is 0.875 (green line denotes the reference line). The results of Table 5 suggest that the proposed model achieves the high precision, F-Score and recall value in comparison with the existing other methods. High true positive rate or precision signifies more accurate results for mitosis detection. In our problem, we only knew the total number of mitosis patches. However, we were unaware of the number of non-mitosis patches in the testing data. Therefore, for testing the number of non-mitosis is considered for almost all non-mitosis portions in the image. The results show the importance of the model along with handcrafted feature, the relatively poor performance of the existing models or models without the handcrafted layer. It is the most interesting result of this work. Usually, people are adopting deep learning to extract features (i.e. without performing manually) automatically. In this case, the use of handcrafted ones is making things better. Table 8 shows the performance comparison table between with and without using handcrafted features. The performance measures without handcrafted features show comparatively lower precision, recall and F-score value in comparison with the proposed method. This table shows that deep learning 


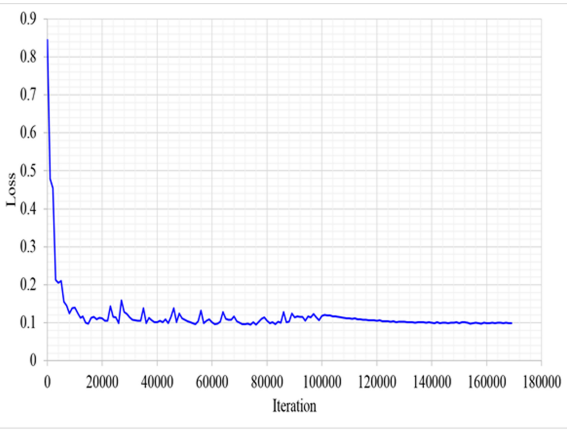

(a)

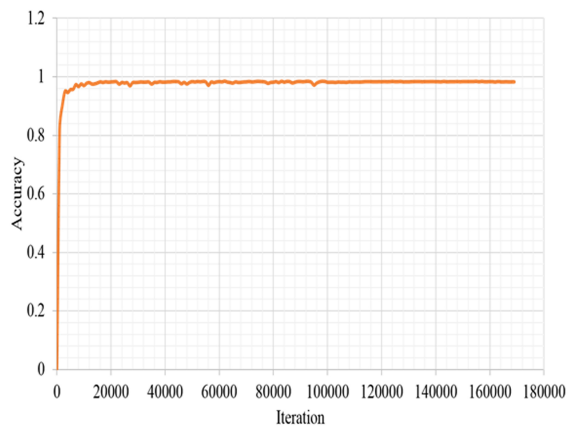

(b)

Figure 12: Training performance graphs: (a) Loss vs iteration graph, (b) accuracy versus iteration graph

with handcrafted features confirms mitosis and non-mitosis more accurately in comparison to the one without handcrafted features.

\subsection{Comparative Results}

We engaged 5-fold cross validation where each subset was included $20 \%$ of the total data. The each fold cross-validation performances has been shown in table 6 . Table 7 shows comparison result among most recently published articles and the proposed model. From this table, it is evident that our model performs satisfactorily regarding $\mathrm{F}$-score, precision and recall value than the existing methods worked on similar kind of dataset. Fig. 11 shows the comparative graphs of F-score value on the MITOS-ATYPIA dataset. Furthermore, performances based on various combinations of training and testing dataset have been discussed in table 9.

\subsection{Computation Time}

In clinical application, computation time is crucial as WSI images consist of thousands of HPFs. The superiority of our proposed model is that it's detection time is very less. It took around 0.3 seconds for each HPF input image patch. Furthermore, the proposed cascaded framework achieved a comparable classification accuracy of $98 \%$. It makes the framework possible for real-world clinical applications. Fig. 12 shows training performance graphs. During training, classification accuracy observed more than $98 \%$ at the 120,000 iterations.

\section{DISCUSSION}

Histopathological images are contaminated with subjectivity and incompleteness. These are unavoidable to morphological variation and a sheer number of mitosis. The architecture presented in this paper on the basis of dataset available in hand. An extensive network requires more training time, more variation in 
the representations of high-level object and expenses for other resources. In the proposed model, morphological, textural and intensity features deliver best results. Selection of input features is very much important because suitable features reduce the time duration, complexity and provide better performance.

Automatic mitosis detection from BC histopathology images could provide better benefits to pathologists in many ways. The most important application is to locate, identify and count all mitosis in HPF images. This technique could facilitate quantitative analysis, reduce intra-observer variability and remove tediousness in routine pathological practice. There has few limitation which has been discussed below:

\subsection{Limitations}

- First, the proposed framework has been trained based on $40 \times$ magnification images. Hence, it may not work properly if lesser or higher magnification images are used.

- Second, the shape of mitotic figures is different in each of the mitosis phases which may increase false positive rate.

- Third, huge datasets are required for training using deep learning. This is one of the main obstacles the researchers may face as there are very limited standard dataset available online.

- Fourth, due to some possible variation in the slide preparation (staining process, concentration, proportion, etc.), pathologists may miss some prominent mitotic figures.

These limitations can be reduced to a greater extent if the image patches are selected with proper concentration and expertise.

\section{CONCLUSION}

The proposed model has been used for automated detection of mitosis in routine H\&E stained BC histology images. The use of deep architecture reinforced with handcrafted features issued from three challenges increased the performance of the proposed deep learning framework. The comparison results show the strength of the proposed methodology. Moreover, the F-score, precision and recall value indicates better performance evaluation results as compared with the other existing techniques. The proposed deep architecture can enable classification of topological features on BC tumour histology. Finally, the presented mitosis detection model could potentially offer a useful tool to pathologists for a better understanding of $\mathrm{BC}$ grading. 


\section{ACKNOWLEDGEMENTS}

We would like to acknowledge Department of Science and Technology (DST), Govt. of India, for providing the INSPIRE fellowship (IVR Number: 201400105113) and Indo-French Centre for Promotion of Advanced Research (CEFIPRA) for Raman-Charpak fellowship 2015 (RCF-IN-0071). C. Chakraborty acknowledges Ministry of Human Resource Development (MHRD), Govt. of India for partial support to carry out this work (grant no: 4-23/2014 T.S.I. date: 14-02-2014).

The major part of this research work has been carried out at Laboratoire d'Imagerie Biomédicale (LIB), University Pierre and Marie Curie (UPMC-Paris 6), Paris, France under the guidance of prof. Daniel Racoceanu. The authors are thankful to the prof. Philippe DUMEE, LIB, (UPMC-Paris 6) for the his helpful support to carry out this research.

\section{References}

[1] M. Saha, I. Arun, B. Basak, S. Agarwal, R. Ahmed, S. Chatterjee, R. Bhargava, C. Chakraborty, Quantitative microscopic evaluation of mucin areas and its percentage in mucinous carcinoma of the breast using tissue histological images, Tissue and Cell 48 (3) (2016) 265-273.

[2] M. Saha, C. Chakraborty, I. Arun, R. Ahmed, S. Chatterjee, An advanced deep learning approach for ki-67 stained hotspot detection and proliferation rate scoring for prognostic evaluation of breast cancer, Scientific Reports 7 (1) (2017) 3213.

[3] E. A. Rakha, J. S. Reis-Filho, F. Baehner, D. J. Dabbs, T. Decker, V. Eusebi, S. B. Fox, S. Ichihara, J. Jacquemier, S. R. Lakhani, et al., Breast cancer prognostic classification in the molecular era: the role of histological grade, Breast Cancer Research 12 (4) (2010) 1.

[4] M. Saha, R. Mukherjee, C. Chakraborty, Computer-aided diagnosis of breast cancer using cytological images: A systematic review, Tissue and Cell 48 (5) (2016) 461-474.

[5] C. W. Elston, I. O. Ellis, Pathological prognostic factors in breast cancer. i. the value of histological grade in breast cancer: experience from a large study with long-term follow-up, Histopathology 19 (5) (1991) 403-410.

[6] D. Mazia, Mitosis and the physiology of cell division, The cell 3 (1961) $77-412$.

[7] R. J. B. King, M. W. Robins, Cancer biology, Pearson Education, 2006.

[8] H. Irshad, S. Jalali, L. Roux, D. Racoceanu, L. J. Hwee, G. Le Naour, F. Capron, et al., Automated mitosis detection using texture, sift features and hmax biologically inspired approach, Journal of Pathology informatics 4 (2) (2013) 12. 
[9] L. Traore, C. Daniel, M.-C. Jaulent, T. Schrader, D. Racoceanu, Y. Kergosien, A sustainable visual representation of available histopathological digital knowledge for breast cancer grading, diagnostic pathology 2 (1).

[10] MITOS-ATYPIA 14 (2014 (accessed October 24, 2016)). URL https://grand-challenge.org/site/mitos-atypia-14/home/

[11] L. Roux, D. Racoceanu, N. Loménie, M. Kulikova, H. Irshad, J. Klossa, F. Capron, C. Genestie, G. Naour, M. Gurcan, Mitosis detection in breast cancer histological images an icpr 2012 contest, Journal of Pathology Informatics 4 (1) (2013) 8. doi:10.4103/2153-3539.112693.

[12] International Conference on Pattern Recognition(ICPR)-2012 (2012 (accessed October 3, 2016)).

URL http://ludo17.free.fr/mitos_2012/dataset.html

[13] A. Paul, D. P. Mukherjee, Mitosis detection for invasive breast cancer grading in histopathological images, IEEE Transactions on Image Processing 24 (11) (2015) 4041-4054.

[14] H. Irshad, et al., Automated mitosis detection in histopathology using morphological and multi-channel statistics features, Journal of pathology informatics 4 (1) (2013) 10.

[15] H. Irshad, A. Gouaillard, L. Roux, D. Racoceanu, Multispectral band selection and spatial characterization: Application to mitosis detection in breast cancer histopathology, Computerized Medical Imaging and Graphics 38 (5) (2014) 390-402.

[16] H. Irshad, A. Gouaillardd, L. Roux, D. Racoceanu, Spectral band selection for mitosis detection in histopathology, in: 2014 IEEE 11th International Symposium on Biomedical Imaging (ISBI), IEEE, 2014, pp. 1279-1282.

[17] S. Huh, R. Bise, M. Chen, T. Kanade, et al., Automated mitosis detection of stem cell populations in phase-contrast microscopy images, IEEE transactions on medical imaging 30 (3) (2011) 586-596.

[18] F. B. Tek, et al., Mitosis detection using generic features and an ensemble of cascade adaboosts, Journal of pathology informatics 4 (1) (2013) 12.

[19] A. Liu, K. Li, T. Kanade, Mitosis sequence detection using hidden conditional random fields, in: 2010 IEEE International Symposium on Biomedical Imaging: From Nano to Macro, IEEE, 2010, pp. 580-583.

[20] M. Schlachter, M. Reisert, C. Herz, F. Schlurmann, S. Lassmann, M. Werner, H. Burkhardt, O. Ronneberger, Harmonic filters for 3d multichannel data: Rotation invariant detection of mitoses in colorectal cancer, IEEE transactions on medical imaging 29 (8) (2010) 1485-1495. 
[21] C. Y. Tao, J. Hoyt, Y. Feng, A support vector machine classifier for recognizing mitotic subphases using high-content screening data, Journal of biomolecular screening 12 (4) (2007) 490-496.

[22] C. Malon, E. Brachtel, E. Cosatto, H. P. Graf, A. Kurata, M. Kuroda, J. S. Meyer, A. Saito, S. Wu, Y. Yagi, Mitotic figure recognition: Agreement among pathologists and computerized detector, Analytical Cellular Pathology 35 (2) (2012) 97-100.

[23] C.-H. Huang, H.-K. Lee, Automated mitosis detection based on exclusive independent component analysis, in: Pattern Recognition (ICPR), 2012 21st International Conference on, IEEE, 2012, pp. 1856-1859.

[24] A. Tashk, M. S. Helfroush, H. Danyali, M. Akbarzadeh, An automatic mitosis detection method for breast cancer histopathology slide images based on objective and pixel-wise textural features classification, in: Information and Knowledge Technology (IKT), 2013 5th Conference on, IEEE, 2013, pp. 406-410.

[25] A. Tashk, M. Helfroush, H. Danyali, M. Akbarzadeh, A novel cad system for mitosis detection using histopathology slide images., Journal of medical signals and sensors 4 (2) (2014) 139-149.

[26] R. Nateghi, H. Danyali, M. SadeghHelfroush, F. P. Pour, Automatic detection of mitosis cell in breast cancer histopathology images using genetic algorithm, in: Biomedical Engineering (ICBME), 2014 21th Iranian Conference on, IEEE, 2014, pp. 1-6.

[27] M. Üstüner, G. Bilgin, Mitosis detection on histopathological images using statistical detection algorithms, in: 2015 23nd Signal Processing and Communications Applications Conference (SIU), IEEE, 2015, pp. 540-543.

[28] A. Giusti, C. Caccia, D. C. Cireşari, J. Schmidhuber, L. M. Gambardella, A comparison of algorithms and humans for mitosis detection, in: 2014 IEEE 11th International Symposium on Biomedical Imaging (ISBI), IEEE, 2014, pp. 1360-1363.

[29] A. S. Tripathi, A. Mathur, M. Daga, M. Kuse, O. C. Au, 2-simdom: a 2-sieve model for detection of mitosis in multispectral breast cancer imagery, in: 2013 IEEE International Conference on Image Processing, IEEE, 2013, pp. $611-615$.

[30] V. Roullier, O. Lézoray, V.-T. Ta, A. Elmoataz, Multi-resolution graph-based analysis of histopathological whole slide images: Application to mitotic cell extraction and visualization, Computerized Medical Imaging and Graphics 35 (7) (2011) 603-615.

[31] H. Sharma, N. Zerbe, S. Lohmann, K. Kayser, O. Hellwich, P. Hufnagl, A review of graph-based methods for image analysis in digital histopathology, Diagnostic Pathology 1 (1). 
[32] K. Kayser, S. Borkenfeld, R. Carvalho, A. Djenouni, G. Kayser, How to analyze structure and function in tissue-based diagnosis?, Diagnostic Pathology 2 (1).

[33] D. C. Cireşan, A. Giusti, L. M. Gambardella, J. Schmidhuber, Mitosis detection in breast cancer histology images with deep neural networks, in: International Conference on Medical Image Computing and Computerassisted Intervention, Springer, 2013, pp. 411-418.

[34] M. Veta, P. J. van Diest, M. Jiwa, S. Al-Janabi, J. P. Pluim, Mitosis counting in breast cancer: Object-level interobserver agreement and comparison to an automatic method, PloS one 11 (8) (2016) e0161286.

[35] M. Veta, P. J. Van Diest, S. M. Willems, H. Wang, A. Madabhushi, A. CruzRoa, F. Gonzalez, A. B. Larsen, J. S. Vestergaard, A. B. Dahl, et al., Assessment of algorithms for mitosis detection in breast cancer histopathology images, Medical image analysis 20 (1) (2015) 237-248.

[36] G. E. Dahl, T. N. Sainath, G. E. Hinton, Improving deep neural networks for lvcsr using rectified linear units and dropout, in: 2013 IEEE International Conference on Acoustics, Speech and Signal Processing, IEEE, 2013, pp. 8609-8613.

[37] J. Masci, U. Meier, D. Cireşan, J. Schmidhuber, Stacked convolutional autoencoders for hierarchical feature extraction, in: International Conference on Artificial Neural Networks, Springer, 2011, pp. 52-59.

[38] Y. Jia, E. Shelhamer, J. Donahue, S. Karayev, J. Long, R. Girshick, S. Guadarrama, T. Darrell, Caffe: Convolutional architecture for fast feature embedding, in: Proceedings of the 22nd ACM international conference on Multimedia, ACM, 2014, pp. 675-678.

[39] K. Sirinukunwattana, S. E. A. Raza, Y.-W. Tsang, D. R. Snead, I. A. Cree, N. M. Rajpoot, Locality sensitive deep learning for detection and classification of nuclei in routine colon cancer histology images, IEEE transactions on medical imaging 35 (5) (2016) 1196-1206.

[40] J. Deng, W. Dong, R. Socher, L.-J. Li, K. Li, L. Fei-Fei, Imagenet: A large-scale hierarchical image database, in: Computer Vision and Pattern Recognition, 2009. CVPR 2009. IEEE Conference on, IEEE, 2009, pp. $248-255$.

[41] M. Saha, S. Agarwal, I. Arun, R. Ahmed, S. Chatterjee, P. Mitra, C. Chakraborty, Histogram based thresholding for automated nucleus segmentation using breast imprint cytology, in: Advancements of Medical Electronics, Springer, 2015, pp. 49-57.

[42] A. Krizhevsky, I. Sutskever, G. E. Hinton, Imagenet classification with deep convolutional neural networks, in: Advances in neural information processing systems, 2012, pp. 1097-1105. 
[43] N. Srivastava, G. E. Hinton, A. Krizhevsky, I. Sutskever, R. Salakhutdinov, Dropout: a simple way to prevent neural networks from overfitting., Journal of Machine Learning Research 15 (1) (2014) 1929-1958.

[44] S. Albarqouni, C. Baur, F. Achilles, V. Belagiannis, S. Demirci, N. Navab, Aggnet: Deep learning from crowds for mitosis detection in breast cancer histology images, IEEE transactions on medical imaging 35 (5) (2016) 1313-1321.

[45] E. Aptoula, N. Courty, S. Lefèvre, Mitosis detection in breast cancer histological images with mathematical morphology, in: Signal Processing and Communications Applications Conference (SIU), 2013 21st, IEEE, 2013, pp. $1-4$.

[46] H. Chang, L. A. Loss, B. Parvin, Nuclear segmentation in h\&e sections via multi-reference graph cut $(\mathrm{mrgc})$, in: International symposium biomedical imaging, 2012.

[47] H. Wang, A. Cruz-Roa, A. Basavanhally, H. Gilmore, N. Shih, M. Feldman, J. Tomaszewski, F. Gonzalez, A. Madabhushi, Cascaded ensemble of convolutional neural networks and handcrafted features for mitosis detection, in: SPIE Medical Imaging, International Society for Optics and Photonics, 2014, pp. 90410B-90410B.

[48] H. Wang, A. CruzRoa, A. Basavanhally, H. Gilmore, N. Shih, M. Feldman, J. Tomaszewski, F. Gonzalez, A. Madabhushi, Mitosis detection in breast cancer pathology images by combining handcrafted and convolutional neural network features., Journal of medical imaging (Bellingham, Wash.) 1 (3) (2014) 034003-034003.

[49] C. D. Malon, E. Cosatto, et al., Classification of mitotic figures with convolutional neural networks and seeded blob features, Journal of pathology informatics 4 (1) (2013) 9.

[50] Assessment of Mitosis Detection Algorithms 2013 (AMIDA13) (2016 (accessed October 24, 2016)).

URL http://amida13.isi.uu.nl/

[51] S.-C. B. Lo, H.-P. Chan, J.-S. Lin, H. Li, M. T. Freedman, S. K. Mun, Artificial convolution neural network for medical image pattern recognition, Neural networks 8 (7) (1995) 1201-1214.

[52] C. A. O'Brien, A. Pollett, S. Gallinger, J. E. Dick, A human colon cancer cell capable of initiating tumour growth in immunodeficient mice, Nature 445 (7123) (2007) 106-110.

[53] A. Uppuluri, Glcm texture features (2008).

URL https://in.mathworks.com/matlabcentral/fileexchange/ 22187-glcm-texture-features 
[54] M. M. R. Krishnan, M. Pal, R. R. Paul, C. Chakraborty, J. Chatterjee, A. K. Ray, Computer vision approach to morphometric feature analysis of basal cell nuclei for evaluating malignant potentiality of oral submucous fibrosis, Journal of medical systems 36 (3) (2012) 1745-1756.

[55] M. M. R. Krishnan, A. Choudhary, C. Chakraborty, A. K. Ray, R. R. Paul, Texture based segmentation of epithelial layer from oral histological images, Micron 42 (6) (2011) 632-641.

[56] Tumor Proliferation Assessment Challenge 2016 (TUPAC16) (2016 (accessed October 24, 2016)).

URL http://tupac.tue-image.nl/

[57] R. C. Gonzalez, R. Woods, S. Eddins, Vol. 2. digital image processing using matlab (2009).

[58] J. Xu, L. Xiang, Q. Liu, H. Gilmore, J. Wu, J. Tang, A. Madabhushi, Stacked sparse autoencoder (ssae) for nuclei detection on breast cancer histopathology images, IEEE transactions on medical imaging 35 (1) (2016) 119-130.

\section{Figure Captions}

1. Figure 1: High power field image (40× magnification) generation

2. Figure 2: RGB to Blue ratio image conversion (a) original RGB image; (b) blue ratio image

3. Figure 3: Flow diagram of the proposed deep learning network

4. Figure 4: Work flow diagram of the handcrafted features generation

5. Figure 5: Flow diagram of the proposed methodology

6. Figure 6: Correlation matrix for 24 global features for image classification

7. Figure 7: Shows filters and learned features maps of the proposed deep network, (a) first layer filter (b) first hidden layer $90(10 \times 9)$ units. (c) second hidden layer $100(10 \times 10)$ units. (d) third hidden layer $100(10 \times 10)$ units. (e) fourth hidden layer $100(10 \times 10)$ units. (f) fifth hidden layer $100(10 \times 10)$

8. Figure 8: The mitosis detection results 
9. Figure 9: The mitosis detection results on HPF images at $40 \times$ magnification, Hamamatsu scanner: (a) original image, (b) final result mitosis (yellow circle) and non-mitosis (green circle); Aperio scanner: (c) original image, (d) final result mitosis (yellow circle) and non-mitosis (green circle)

10. Figure 10: ROC curve

11. Figure 11: Comparison of F-score values using various methods on MITOSATYPIA-14 dataset

12. Figure 12: Training performance graphs: (a) Loss versus iteration graph, (b) accuracy versus iteration graph

\section{Table Captions}

1. Table 1. Categorization of mitosis detection approaches

2. Table 2.Comparative results of different dataset and methods

3. Table 3. The 55 Hand-crafted features extracted from the mitosis and non-mitosis image patches

4. Table 4. Proposed deep learning architecture

5. Table 5. Performance measures for mitosis detection

6. Table 6. 5-fold cross-validation

7. Table 7. Comparison with other methodologies

8. Table 8. Performance with/without handcrafted features

9. Table 9. Performance based on various combinations of training and testing dataset 